\title{
Blood Gases during Cardiopulmonary Resuscitation in Predicting Arrest Cause between Primary Cardiac Arrest and Asphyxial Arrest
}

\author{
Sei Jong Bae, M.D., Byung Kook Lee, M.D., Ki Tae Kim, M.D., Kyung Woon Jeung, M.D., \\ Hyoung Youn Lee, M.D., Yong Hun Jung, M.D., Geo Sung Lee, M.D., \\ Sun Pyo Kim, M.D.* and Seung Joon Lee, M.D. ${ }^{\dagger}$
}

Department of Emergency Medicine, Chonnam National University Hospital, *Department of Emergency Medicine,

Chosun University Hospital, Gwangju, ${ }^{\dagger}$ Department of Emergency Medicine, Seoul St. Mary's Hospital, Seoul, Korea

\begin{abstract}
Background: If acid-base status and electrolytes on blood gases during cardiopulmonary resuscitation (CPR) differ between the arrest causes, this difference may aid in differentiating the arrest cause. We sought to assess the ability of blood gases during CPR to predict the arrest cause between primary cardiac arrest and asphyxial arrest.

Methods: A retrospective study was conducted on adult out-of-hospital cardiac arrest patients for whom blood gas analysis was performed during CPR on emergency department arrival. Patients were divided into two groups according to the arrest cause: a primary cardiac arrest group and an asphyxial arrest group. Acid-base status and electrolytes during CPR were compared between the two groups.

Results: Presumed arterial samples showed higher potassium in the asphyxial arrest group $(\mathrm{p}<0.001)$. On the other hand, presumed venous samples showed higher potassium $(\mathrm{p}=0.001)$ and $\mathrm{PCO}_{2}(\mathrm{p}<0.001)$ and lower $\mathrm{pH}$ $(\mathrm{p}=0.008)$ and oxygen saturation $(\mathrm{p}=0.01)$ in the asphyxial arrest group. Multiple logistic regression analyses revealed that arterial potassium (OR 5.207, 95\% CI $1.430-18.964, \mathrm{p}=0.012)$ and venous $\mathrm{PCO}_{2}(\mathrm{OR} 1.049,95 \% \mathrm{CI}$ $1.021-1.078, \mathrm{p}<0.001)$ were independent predictors of asphyxial arrest. Receiver operating characteristic curve analyses indicated an optimal cut-off value for arterial potassium of $6.1 \mathrm{mEq} / \mathrm{L}$ (sensitivity $100 \%$ and specificity 86.4\%) and for venous $\mathrm{PCO}_{2}$ of $70.9 \mathrm{mmHg}$ (sensitivity $84.6 \%$ and specificity $65.9 \%$ ).

Conclusions: The present study indicates that blood gases during CPR can be used to predict the arrest cause. These findings should be confirmed through further studies.
\end{abstract}

Key Words: asphyxia, blood gas analysis, cardiopulmonary resuscitation, heart arrest, potassium.

\section{INTRODUCTION}

Cardiac arrest and resulting cessation of respiration and blood flow cause a shift from aerobic to anaerobic metabolism. During cardiac arrest, intense acidosis at the tissue level occurs from the accumulation of end products such as carbon dioxide, lactate, and hydrogen ions. Even when cardiopulmonary resuscitation (CPR) is applied, the blood flow generated rarely exceeds $20 \%$ to $30 \%$ of normal blood flow and fails to meet metabolic requirements.[1,2] As a result, tissue acidosis persists

Received on September 14, 2012, Revised on January 24, 2013 (1st), February 1, 2013 (2nd), Accepted on February 4, 2013

Correspondence to: Kyung Woon Jeung, Department of Emergency Medicine, Chonnam National University Hospital, 671, Jebong-ro, Dong-gu, Gwangju 501-757, Korea Tel: 062-220-6809, Fax: 062-228-7417

E-mail: neoneti@hanmail.net during the CPR period. In addition to the tissue acidosis, electrolyte imbalances such as hyperkalemia can occur during the cardiac arrest and the CPR period. During these periods, serum potassium concentrations may increase because of the tissue acidosis and subsequent release of intracellular potassium into the systemic circulation. Several animal studies have shown that both ischemic and hypoxic insults also cause potassium release from cells.[3,4] Acidosis and hyperkalemia not only complicate cardiac arrest, but also, they cause cardiac arrest. The present CPR guideline emphasises the importance of diagnosing and treating the underlying causes of the cardiac arrest such as acidosis and hyperkalemia.[5] To identify treatable causes rapidly, blood gas analysis is widely used during CPR.

The two most prevalent causes of cardiac arrest are primary cardiac disease and asphyxia. Primary cardiac disease including malignant dysrhythmia or coronary artery disease is the leading 
cause of sudden cardiac arrest. Cardiac arrest secondary to the primary cardiac disease develops most commonly with sudden onset of ventricular fibrillation (VF). Asphyxia is the most common cause of cardiac arrest in children. In adults, respiratory failure from various causes frequently leads to asphyxial arrest if it is not treated adequately. Asphyxia, in contrast to sudden VF cardiac arrest, causes hypoxia, hypercarbia, and hypotension for variable durations prior to the onset of cardiac arrest. Thus, acidosis and electrolyte imbalances during cardiac arrest and CPR would be worse in asphyxial arrest than in primary cardiac arrest. A number of previous studies have reported blood gas findings during CPR. However, most of them have included only cardiac arrest caused by the primary cardiac disease.[6-10] To our knowledge, no prior study has assessed differences in blood gases during CPR between the causes of cardiac arrest. If acid-base status and electrolytes on blood gases during CPR differ between the causes of cardiac arrest, this difference, together with other clinical findings, may aid in differentiating the cause of cardiac arrest.

In this retrospective study, we compared acid-base status and electrolytes in blood gas samples obtained during CPR between primary cardiac arrest and asphyxial arrest in out-of-hospital cardiac arrest patients. We sought to assess the ability of blood gases during CPR to predict the cause of cardiac arrest between primary cardiac arrest and asphyxial arrest.

\section{MATERIALS AND METHODS}

This study was approved by the Institutional Review Board of Chonnam National University Hospital, Gwangju, Korea. This was a retrospective study of non-traumatic out-of-hospital cardiac arrest patients who presented at the emergency department (ED) of Chonnam National University Hospital and Chosun University Hospital between January 2006 and December 2010.

In both hospitals, resuscitation procedures were performed by emergency physicians in accordance with the present CPR guidelines.[5] Samples for blood gas analyses during CPR were obtained based on the decision by an attending emergency physician, and were taken from a femoral vessel in an attempt to take the sample from the femoral artery. Blood samples were immediately analysed using a blood gas machine (RapidLab865, Bayer Health Care, Fernwald, Germany).

We included patients meeting the following criteria: (1) age $\geq 18$ years; (2) non-traumatic cardiac arrest. The exclusion criteria were as follows: no identifiable cause of cardiac arrest, no available blood gas results obtained during CPR, and missing medical records. Patients who met the inclusion/exclusion criteria were identified using electronic medical records, and were divided into two groups according to the cause of cardiac arrest: a primary cardiac arrest group and an asphyxial arrest group. The primary cardiac arrest group included patients who suffered from sudden cardiac arrest caused by acute coronary syndrome or malignant dysrhythmia. The asphyxial arrest group included patients who suffered from arrest secondary to respiratory failure.

The following variables were obtained for each patient: age; sex; pre-existing disease; presence of a witness on collapse; initial cardiac rhythm at the ED; duration of CPR; ROSC; elapsed time from collapse to ED visit; discharge outcome; cause of arrest; blood gas obtained during CPR; elapsed time between ED arrival and sampling for blood gas analysis; and amounts of epinephrine and sodium bicarbonate administered between ED arrival and sampling for blood gas analysis. It is often difficult to distinguish which sample was actually obtained from femoral vessels between arterial and venous blood during CPR.[11] Based on previous studies, blood samples were considered consistent with arterial sampling when $\mathrm{PO}_{2}$ exceeded $50 \mathrm{mmHg}$ and, were considered consistent with venous sampling when $\mathrm{PO}_{2}$ was less than $50 \mathrm{mmHg}$.[7,12] In a study by Langhelle et al.[12], which included 17 cardiac arrest patients who were intubated and mechanically ventilated 12/ min with $100 \%$ oxygen during $\mathrm{CPR}$, the lowest arterial $\mathrm{PO}_{2}$ was $54 \mathrm{mmHg}$. Steedman and Robertson [7] observed large differences between arterial and central venous $\mathrm{PO}_{2}$ in a study which compared simultaneously measured arterial and central venous blood gases during CPR. They suggested that intermittent percutaneous femoral sampling can be satisfactorily used for study purposes allowing differentiation of arterial from venous blood on the basis of $\mathrm{PO}_{2}$.

Data were analysed using SPSS 15.0 (Chicago, IL, USA). Continuous variables were investigated for normality using the Shapiro-Wilk test. Normally distributed continuous variables were summarised as means and standard deviations (SD). The independent $\mathrm{t}$ test was conducted to compare between the two groups. Non-normally distributed continuous variables were summarised as medians with interquartile ranges and the non-parametric Mann-Whitney U test was conducted. Categorical variables were summarised as frequencies and percentages. The chi-square test or the Fisher's exact test was used where appropriate. Logistic regression analyses were undertaken to identify independent predictors of asphyxial arrest between primary 
cardiac arrest and asphyxial arrest. Significant variables from univariate analyses $(\mathrm{p}<0.10)$ were entered into the model. The discriminative power of the independent predictors from the logistic regression was evaluated by Receiver Operating Characteristic (ROC) analysis using the Medcalc program (Medcalc Software, Mariakerke, Belgium). Area Under the Curve (AUC) was given with $95 \%$ confidence intervals. A value of $\mathrm{p}<0.05$ was considered statistically significant.

\section{RESULTS}

In both hospitals, during the study period, 506 adult nontraumatic out-of-hospital cardiac arrest patients visited the ED and received CPR. Of the 506, 116 who had cardiac arrest of unknown aetiology, 278 patients in whom blood gas analysis was not performed during CPR, and 4 patients whose medical records were missing were excluded, leaving 108 eligible patients: 74 in the primary cardiac arrest group and 34 patients in the asphyxial arrest group (Fig. 1). The causes of asphyxial arrest were severe acute respiratory failure from lung diseases in 13 patients, hanging in 9 patients, massive hemoptysis in 6 patients, foreign body airway obstruction in 3 patients, respiratory failure from amyotrophic lateral sclerosis in 2 patients, and drowning in one patient.

Table 1 shows the clinical characteristics for all 108 patients. The number of cases where cardiac arrest was witnessed was significantly higher in the primary cardiac arrest group ( $p$ $=0.001$ ), and the initial cardiac rhythm was significantly different between the two groups $(p=0.002)$. However, no sig- nificant differences between the two groups were found for age, sex, pre-existing disease, rate of ROSC, elapsed time from collapse to ED visit, and discharge outcome. The elapsed time between ED arrival and sampling for blood gas analysis was 6 $(4-10) \mathrm{min}$. The amounts of epinephrine and sodium bicarbonate administered between ED arrival and sampling for blood gas analysis were $2.5(1.0-4.0) \mathrm{mg}$ and $0(0-3) \mathrm{mEq}$, respectively. The elapsed time and the amounts of epinephrine and sodium bicarbonate administered between ED arrival and sampling for blood gas analysis were not different between the two groups.

On the basis of $\mathrm{PO}_{2}$, blood gas analyses in 38 patients (35.2\%) were considered to be arterial; in the remaining 70 patients $(64.8 \%)$ they were considered to be venous. Blood gas variables in presumed arterial and venous blood samples are shown in Table 2. In the presumed arterial samples, only potassium showed a statistically significant difference between the two groups among the blood gas variables $(\mathrm{p}<0.001)$. $\mathrm{PCO}_{2}$ was higher and oxygen saturation was lower in the asphyxial arrest group, although the differences were not significant. $\mathrm{pH}$, $\mathrm{PO}_{2}$, base excess, and bicarbonate were comparable between the two groups. Differences between the two groups were more prominent in the presumed venous samples. $\mathrm{pH}(\mathrm{p}=$ 0.008 ) and oxygen saturation ( $\mathrm{p}=0.01)$ were significantly lower and $\mathrm{PCO}_{2}(\mathrm{p}<0.001)$ and potassium $(\mathrm{p}=0.001)$ were higher in the asphyxial arrest group compared with the primary cardiac arrest group.

In multiple logistic regression analyses, potassium was an independent predictor of asphyxial arrest between primary cardiac

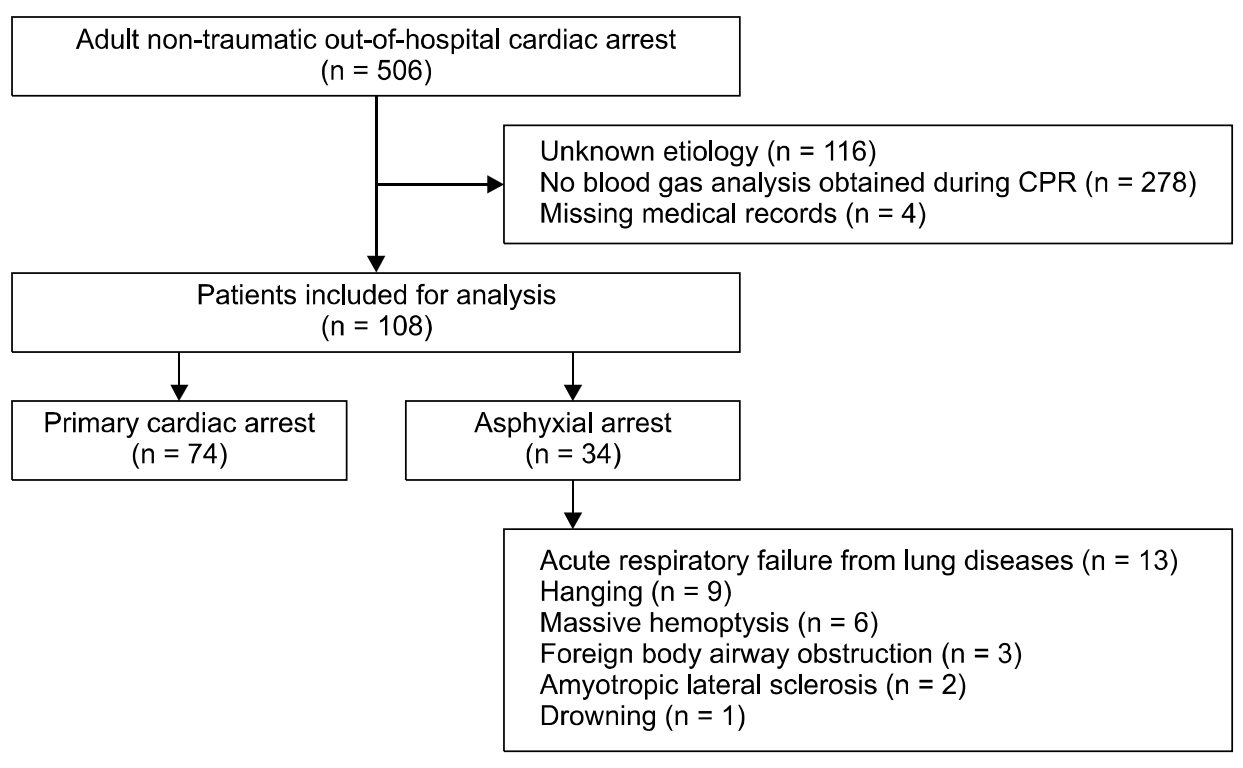

Fig. 1. Study participants. 
Table 1. Clinical Characteristics of Patients in the Present Study

\begin{tabular}{|c|c|c|c|}
\hline & $\begin{array}{l}\text { Primary cardiac arrest } \\
\qquad(\mathrm{n}=74)\end{array}$ & $\begin{array}{l}\text { Asphyxial cardiac arrest } \\
\qquad(\mathrm{n}=34)\end{array}$ & $\mathrm{p}$ value \\
\hline Age, years & $68.0(57.8-75.0)$ & $69.0(58.5-73.3)$ & 0.771 \\
\hline Male gender, n (\%) & $60(81.1)$ & $23(67.7)$ & 0.145 \\
\hline \multicolumn{4}{|l|}{ Preexisting disease, $\mathrm{n}(\%)$} \\
\hline Diabetes & $19(25.7)$ & $4(11.8)$ & 0.216 \\
\hline Hypertension & $36(48.6)$ & $9(26.5)$ & 0.072 \\
\hline Renal disease & $2(2.7)$ & $0(0.0)$ & 0.773 \\
\hline Witnessed collapse, n (\%) & $61(83.6)$ & $18(52.9)$ & 0.001 \\
\hline Presenting rhythm, n (\%) & & & 0.002 \\
\hline $\mathrm{VF} /$ pulseless VT & $20(27.0)$ & $0(0.0)$ & \\
\hline PEA & $5(6.8)$ & $2(5.9)$ & \\
\hline Asystole & $31(41.9)$ & $20(58.8)$ & \\
\hline Unknown & $18(24.3)$ & $12(35.3)$ & \\
\hline ROSC, n (\%) & $33(44.6)$ & $17(50.0)$ & 0.601 \\
\hline Elapsed time from collapse to ED visit, $\min ^{\mathrm{a}}$ & $20(15-29)$ & $24(20-30)$ & 0.186 \\
\hline Elapsed time between ED arrival and blood gas sampling, min & $5.0(3.8-10.0)$ & $7.0(5.0-10.3)$ & 0.098 \\
\hline $\begin{array}{l}\text { Amount of epinephrine administered between ED arrival and blood gas } \\
\text { sampling, mg }\end{array}$ & $2(1-4)$ & $3(2-5)$ & 0.605 \\
\hline $\begin{array}{l}\text { Amount of sodium bicarbonate administered between ED arrival and blood } \\
\text { gas sampling, } \mathrm{mEq}\end{array}$ & $0(0-60)$ & $0(0-40)$ & 0.631 \\
\hline Discharge outcome, n (\%) & & & 0.313 \\
\hline Survival discharge & $6(8.1)$ & $1(2.9)$ & \\
\hline Death & $64(86.5)$ & $28(82.4)$ & \\
\hline Transfer to other hospital & $3(4.1)$ & $4(11.8)$ & \\
\hline Hopeless discharge & $1(1.4)$ & $1(2.9)$ & \\
\hline
\end{tabular}

${ }^{a}$ Elapsed time from collapse to ED visit was available in 45 patients in the primary cardiac arrest group and 11 patients in the asphyxial arrest group, respectively. Data are shown as $\mathrm{n}$ (\%) or median (interquartile). VF: ventricular fibrillation; VT: ventricular tachycardia; PEA: pulseless electrical activity; ROSC: restoration of spontaneous circulation; ED: emergency department.

Table 2. Blood Gases in Patients of the Present Study

\begin{tabular}{lcccccc}
\hline \hline & \multicolumn{3}{c}{ Presumed arterial blood } & \multicolumn{3}{c}{ Presumed venous blood } \\
\cline { 2 - 7 } & $\begin{array}{c}\text { Primary cardiac arrest } \\
(\mathrm{n}=30)\end{array}$ & $\begin{array}{c}\text { Asphyxial cardiac arrest } \\
(\mathrm{n}=8)\end{array}$ & $\mathrm{p}$ value & $\begin{array}{c}\text { Primary cardiac arrest } \\
(\mathrm{n}=44)\end{array}$ & $\begin{array}{c}\text { Asphyxial cardiac arrest } \\
(\mathrm{n}=26)\end{array}$ & $\mathrm{p}$ value \\
\hline $\mathrm{pH}$ & $7.084(7.006-7.222)$ & $6.949(6.744-7.136)$ & 0.104 & $7.071 \pm 0.164$ & $6.935 \pm 0.247$ & 0.008 \\
$\mathrm{PCO}_{2}(\mathrm{mmHg})$ & $42.5(27.4-55.7)$ & $59.2(51.0-83.4)$ & 0.050 & $64.8(51.8-83.1)$ & $95.3(79.3-122.0)$ & $<0.001$ \\
$\mathrm{PO}_{2}(\mathrm{mmHg})$ & $70.1(58.6-83.9)$ & $70.1(57.0-84.1)$ & 0.902 & $24.6(14.9-32.7)$ & $17.6(8.4-31.6)$ & 0.073 \\
$\mathrm{Na}(\mathrm{mEq} / \mathrm{L})^{\mathrm{a}}$ & $139(135-141)$ & $139(134-141)$ & 0.892 & $140.3 \pm 5.8$ & $139.2 \pm 9.7$ & 0.390 \\
$\mathrm{~K}(\mathrm{mEq} / \mathrm{L})^{\mathrm{a}}$ & $5.2(4.2-5.5)$ & $8.6(7.9-9.7)$ & $<0.001$ & $4.4(3.7-5.7)$ & $6.6(5.2-8.0)$ & 0.001 \\
$\mathrm{Cl}(\mathrm{mEq} / \mathrm{L})^{\mathrm{a}}$ & $105(103-107)$ & $102(97-104)$ & 0.097 & $103(101-106)$ & $100(96-107)$ & 0.596 \\
$\mathrm{Base} \mathrm{excess} \mathrm{(mmol/L)}$ & $-17.9(-19.3--10.8)$ & $-20.5(-25.0--12.2)$ & 0.314 & $-10.98 \pm 7.12$ & $-11.43 \pm 10.91$ & 0.836 \\
$\mathrm{HCO}_{3}{ }^{-}(\mathrm{mmol} / \mathrm{L})$ & $13.5(10.0-17.1)$ & $11.5(8.0-18.3)$ & 0.712 & $18.76 \pm 4.80$ & $20.52 \pm 6.89$ & 0.212 \\
$\mathrm{O}_{2}$ sat $(\%)$ & $88.7(79.4-93.4)$ & $73.1(63.0-89.5)$ & 0.073 & $26.3(12.9-45.0)$ & $15.9(7.8-27.2)$ & 0.010 \\
\hline
\end{tabular}

${ }^{\text {a }}$ Plasma electrolyte measurements using blood gas analysis samples were performed in 22 patients in the primary cardiac arrest group and 6 patients in the asphyxial arrest group in the presumed arterial blood samples, and were performed in 34 patients in the primary cardiac arrest group and 18 patients in the asphyxial arrest group in the presumed venous blood samples. Data are shown as mean \pm standard deviation or median (interquartile range). $\mathrm{PCO}_{2}$ : partial pressure of carbon dioxide; $\mathrm{PO}_{2}$ : partial pressure of oxygen; $\mathrm{HCO}_{3}{ }^{-}:$bicarbonate; $\mathrm{O}_{2}$ sat: oxygen saturation. 
Table 3. Factors Predicting Asphyxial Arrest between Primary Cardiac Arrest and Asphyxial Arrest

\begin{tabular}{|c|c|c|c|c|}
\hline & \multicolumn{2}{|c|}{ Univariate } & \multicolumn{2}{|l|}{ Mutivariate } \\
\hline & OR $(95 \% \mathrm{CI})$ & $\mathrm{p}$ value & OR $(95 \%$ CI $)$ & $\mathrm{p}$ value \\
\hline \multicolumn{5}{|l|}{ Arterial } \\
\hline $\mathrm{K}(\mathrm{mEq} / \mathrm{L})$ & $5.312(1.461-19.315)$ & 0.011 & $5.091(1.372-18.890)$ & 0.015 \\
\hline \multicolumn{5}{|l|}{ Venous } \\
\hline $\mathrm{pH}$ & $0.032(0.002-0.498)$ & 0.014 & $421.9(0.095-1866681.4)$ & 0.158 \\
\hline $\mathrm{PCO}_{2}(\mathrm{mmHg})$ & $1.050(1.022-1.079)$ & $<0.001$ & $1.062(1.006-1.121)$ & 0.029 \\
\hline $\mathrm{K}(\mathrm{mEq} / \mathrm{L})$ & $1.935(1.221-3.066)$ & 0.005 & $1.546(0.843-2.836)$ & 0.159 \\
\hline $\mathrm{O}_{2}$ sat $(\%)$ & $0.968(0.938-0.999)$ & 0.040 & $0.991(0.950-1.034)$ & 0.683 \\
\hline
\end{tabular}

$\mathrm{PCO}_{2}$ : partial pressure of carbon dioxide; $\mathrm{O}_{2}$ sat: oxygen saturation; OR: odd ratio; CI: confidence interval.

Table 4. AUC Values and Cutoff Values with Highest Combined Sensitivity and Specificity Predicting Asphyxial Arrest in the Present Study

\begin{tabular}{lcccc}
\hline \hline \multicolumn{1}{c}{ Cutoff value } & Sensitivity $(\%)$ & Specificity (\%) & AUC (95\% CI) & p value \\
\hline $\begin{array}{c}\text { Blood gas in the presumed arterial sample } \\
6.1 \mathrm{mEq} / \mathrm{l}\end{array}$ & 100 & 86.4 & $0.973(0.831-1.000)$ & $<0.001$ \\
$\mathrm{~K}$ & & & & \\
$\begin{array}{l}\text { Blood gas in the presumed venous sample } \\
\mathrm{PCO}_{2}\end{array}$ & 84.6 & 65.9 & $0.805(0.693-0.890)$ & $<0.001$ \\
\hline
\end{tabular}

AUC: area under the receiver operating characteristic curve; $\mathrm{CI}$ : confidence interval; $\mathrm{PCO}_{2}$ : partial pressure of carbon dioxide.

A

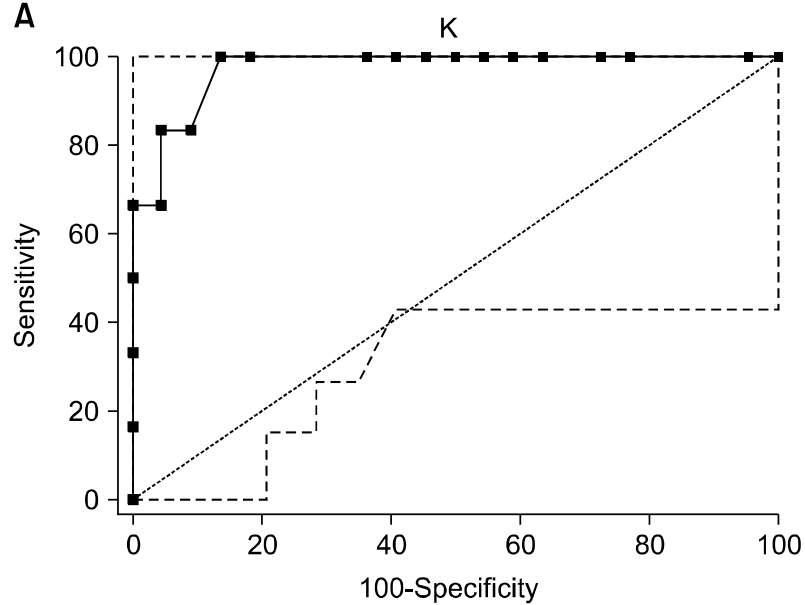

B

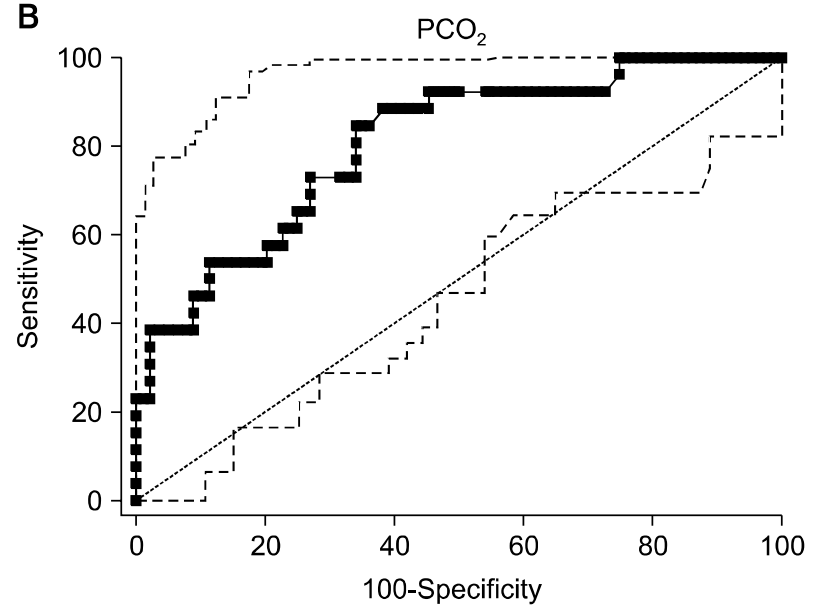

Fig. 2. Receiver operating characteristic curves for (A) arterial potassium and (B) venous $\mathrm{PCO}_{2}$. Solid line indicates asphyxial arrest, and dashed line indicates primary cardiac arrest.

arrest and asphyxial arrest in the presumed arterial sample, with an adjusted odds ratio (OR) of 5.091 (95\% CI 1.372$18.890, \mathrm{p}=0.015$ ) (Table 3 ). In the presumed venous sample, $\mathrm{PCO}_{2}$ was an independent predictor of asphyxial arrest, with an adjusted OR of 1.062 (95\% CI 1.006-1.121, p = 0.029) (Table 3). Cutoff values resulting in highest combined sensitivity and specificity in predicting asphyxial arrest are presented in Table 4. ROC curves for arterial potassium and venous $\mathrm{PCO}_{2}$ are presented in Fig. 2.

\section{DISCUSSION}

In this study, which compared blood gases obtained during CPR between primary cardiac arrest and asphyxial arrest, potassium in the presumed arterial blood and $\mathrm{PCO}_{2}$ in the presumed venous blood were independent predictors of asphyxial arrest between primary cardiac arrest and asphyxial arrest.

Respiratory acidosis represents an important component of tissue acidosis during CPR, and central venous blood gases are 
known to reflect tissue acid-base status more accurately than arterial blood gases during CPR.[8] Although we could not compare blood gases between arterial and venous blood samples, respiratory acidosis was more profound in the presumed venous sample than in the presumed arterial sample regardless of the study group. Several previous studies also reported substantially higher carbon dioxide in the central venous blood compared with the arterial blood during CPR.[6-8] The marked increase in venous $\mathrm{PCO}_{2}$ is attributed to decreased pulmonary excretion of $\mathrm{CO}_{2}$ following a large reduction in pulmonary blood flow during CPR. On the other hand, marked reduction in pulmonary blood flow results in a large increase in the ventilation/perfusion ratio, which leads to relatively low arterial $\mathrm{PCO}_{2}$. In both groups of this study, metabolic acidosis was more profound in the presumed arterial sample than in the presumed venous sample, although the primary acid-base disturbance in the asphyxial arrest group was respiratory acidosis. A similar pattern of acid-base status was observed in a study by Steedman and Robertson.[7] They observed mixed acidbase disturbances inclined towards a metabolic acidosis in arterial blood while simultaneously obtained venous blood revealed profound respiratory acidosis.

At present, the reasons why $\mathrm{PCO}_{2}$ in the presumed venous blood was significantly higher in the asphyxial arrest group are not clear. In a study by Lah et al.[13] which evaluated patterns of end-tidal carbon dioxide $\left(\mathrm{EtCO}_{2}\right)$ in primary cardiac arrest and asphyxial arrest patients, $\mathrm{EtCO}_{2}$ was significantly higher in the asphyxial arrest group compared with the primary cardiac arrest group. The $\mathrm{EtCO}_{2}$ values in the asphyxial arrest group did not show a significant association with ROSC in contrast to $\mathrm{EtCO}_{2}$ values in the primary cardiac arrest group. $\mathrm{EtCO}_{2}$ during $\mathrm{CPR}$ has been shown to have significant correlations with cardiac output, coronary perfusion pressure, and ROSC.[14,15] These studies suggest that the difference in $\mathrm{PCO}_{2}$ in the presumed venous samples between the two groups was not likely due to differences in CPR-generated pulmonary blood flow between the two groups. One possible explanation is hypercarbia preceding the onset of asphyxial arrest. Contrary to VF cardiac arrest, which is a common form of primary cardiac arrest, carbon dioxide accumulated prior to the onset of pulselessness in the asphyxial arrest. Thus, hypercarbia preceding the asphyxial arrest may have been reflected in the blood gases obtained during CPR.

Interestingly, plasma potassium was significantly increased in the asphyxial arrest group in this study. Epinephrine and sodium bicarbonate, which are commonly administered during re- suscitation procedures, can decrease plasma potassium by driving potassium into cells. However, it is unlikely that the relatively normal plasma potassium in the primary cardiac arrest group is associated with epinephrine and sodium bicarbonate administration during CPR because the amounts of epinephrine and sodium bicarbonate administered between ED arrival and sampling for blood gas analysis were not different between the two groups. Potassium efflux from cells can occur as a response to acidosis. A relation between potassium shift and $\mathrm{pH}$ such that serum potassium changes inversely by $0.6 \mathrm{mEq} / \mathrm{L}$ for every 0.1 unit change of $\mathrm{pH}$ is well known.[16] In this study, $\mathrm{pH}$ in the presumed arterial blood was comparable between the two groups. In the presumed venous blood, the difference in plasma potassium between the two groups was too big to be explained by the acidosis-induced shift of intracellular potassium since the difference in $\mathrm{pH}$ between the two groups was about 0.136. Although the mechanism is not clear, it can be speculated that potassium efflux from cells during asphyxia preceding the arrest onset is associated with a relative hyperkalemia during CPR in the asphyxial arrest group. Several studies have found that plasma potassium rises during hypoxia.[17] In a study by Waters et al.,[17] which induced hypoxia in rabbits, potassium concentrations increased by about $50 \%$ compared with baseline potassium concentrations. Ischemia has also been reported to cause efflux of cellular potassium.[18] The proposed mechanism of potassium efflux from cells caused by hypoxia and ischemia is failure of the energy-dependent sodium-potassium pump caused by cellular adenosine triphosphate depletion.

In out-of-hospital cardiac arrest patients, the identification of the arrest cause is difficult, especially during the early minutes of CPR. If rescuers identify hyperkalemia on blood gas analysis during CPR in an asphyxial arrest patient without knowing the arrest cause, they may administer calcium or sodium bicarbonate, considering hyperkalemia as an arrest cause because there is no definite criterion differentiating hyperkalemia as an arrest cause and hyperkalemia complicated by cardiac arrest itself. There are few data to support administration of calcium and/or sodium bicarbonate during CPR. A wide variety of adverse effects, such as hypernatremia, hyperosmolarity, and intracellular acidosis, have been linked to administration of bicarbonate during CPR.[19,20] Most studies on bicarbonate use during CPR showed no benefit or poor outcomes.[21,22] Several researchers have studied effects of calcium administration during CPR. However, most of them could not demonstrate a definite benefit of calcium on survival.[23,24] Based 
on these studies, at present, routine administration of sodium bicarbonate and/or calcium is not recommended for out-of-hospital cardiac arrest patients except for special situations such as cardiac arrest caused by hyperkalemia.[5] Several previous studies have indicated that reoxygenation and high-quality CPR for rapid ROSC, rather than drugs such as sodium bicarbonate and calcium, are the mainstays of treating acidosis and hyperkalemia that are complicating cardiac arrest.[17,25] To avoid unnecessary administration of calcium and/or sodium bicarbonate for hyperkalemia complicated by cardiac arrest, a criterion differentiating hyperkalemia as an arrest cause and hyperkalemia complicated by cardiac arrest should be developed in the future.

The present study has several limitations due to inherent characteristics of its retrospective design. First, the study population consisted only of patients whose arrest cause was primary cardiac disease or asphyxia, and excluded patients suffering cardiac arrest of unknown aetiology. Therefore, our results might not be applicable to all out-of-hospital cardiac arrest patients. In unwitnessed arrest patients who fail to achieve spontaneous circulation, the arrest cause is not identified even after the resuscitation procedure in a considerable number of cases. In the present study, the arrest cause could not be identified in $22.9 \%$ of patients whose medical records were initially reviewed despite in-depth review of medical records. Second, acid-base and electrolytes during cardiac arrest and CPR can be affected by multiple factors including the duration of cardiac arrest and the level of blood flow generated during CPR. However, these factors were not evaluated in this study. Third, the values of potassium in this study depended not only from potassium release during cardiac arrest but also from the basal value before the cardiac arrest. However, we could not obtain the basal potassium values. Fourth, obtaining blood sample during emergency situation might have led to hemolysis and contributed to hyperkalemia. Fifth, blood gas analysis during CPR was obtained based on the decision by an attending emergency physician. In this study, blood gas analysis was not performed during CPR in $54.9 \%$ of the patients whose medical records were initially reviewed. Thus, this study has a potential for ascertainment bias. Sixth, the relatively small sample size may result in low power. Thus, our data must be viewed as hypothesis generating rather than conclusive.

In this study, plasma potassium in the presumed arterial blood and $\mathrm{PCO}_{2}$ in the presumed venous blood predicted asphyxial arrest independently between primary cardiac arrest and asphyxial arrest. Although the findings of this study might not be applicable to all out-of-hospital cardiac arrest patients because of the limitations described above, the present study indicates that blood gases during CPR may be useful in predicting the cause of cardiac arrest. Therefore, our results should be confirmed through further studies.

\section{REFERENCES}

1) Ditchey RV, Winkler JV, Rhodes CA: Relative lack of coronary blood flow during closed-chest resuscitation in dogs. Circulation 1982; 66: 297-302.

2) Rudikoff MT, Maughan WL, Effron M, Freund P, Weisfeldt ML: Mechanisms of blood flow during cardiopulmonary resuscitation. Circulation 1980; 61: 345-52.

3) Cummings JR: Electrolyte changes in heart tissue and coronary arterial and venous plasma following coronary occlusion. Circ Res 1960; 8: 865-70.

4) Conn HL Jr: Effects of digitalis and hypoxia on potassium transfer and distribution in the dog heart. Am J Physiol 1956; 184: 548-52.

5) 2005 American Heart Association guidelines for cardiopulmonary resuscitation and emergency cardiovascular care. Part 7.2: Management of cardiac arrest. Circulation 2005; 112: IV58-66.

6) Adrogué HJ, Rashad MN, Gorin AB, Yacoub J, Madias NE: Assessing acid-base status in circulatory failure. Differences between arterial and central venous blood. $\mathrm{N}$ Engl J Med 1989; 320: 1312-6.

7) Steedman DJ, Robertson CE: Acid base changes in arterial and central venous blood during cardiopulmonary resuscitation. Arch Emerg Med 1992; 9: 169-76.

8) Weil MH, Rackow EC, Trevino R, Grundler W, Falk JL, Griffel MI: Difference in acid-base state between venous and arterial blood during cardiopulmonary resuscitation. N Engl J Med 1986; 315: 1616-8.

9) Pytte M, Dorph E, Sunde K, Kramer-Johansen J, Wik L, Steen PA: Arterial blood gases during basic life support of human cardiac arrest victims. Resuscitation 2008; 77: 35-8.

10) Makino J, Uchino S, Morimatsu H, Bellomo R: A quantitative analysis of the acidosis of cardiac arrest: a prospective observational study. Crit Care 2005; 9: R357-62.

11) Henneman PL, Gruber JE, Marx JA: Development of acidosis in human beings during closed-chest and open-chest CPR. Ann Emerg Med 1988; 17: 672-5.

12) Langhelle A, Sunde K, Wik L, Steen PA: Arterial blood-gases with 500- versus 1000-ml tidal volumes during out-of-hospital CPR. Resuscitation 2000; 45: 27-33.

13) Lah K, Križarić $M$, Grmec S: The dynamic pattern of end-tidal carbon dioxide during cardiopulmonary resuscitation: difference between asphyxial cardiac arrest and ventricular fibrillation/pulseless ventricular tachycardia cardiac arrest. Crit Care 2011; 15: R13.

14) Sanders AB, Atlas M, Ewy GA, Kern KB, Bragg S: Expired 
$\mathrm{PCO} 2$ as an index of coronary perfusion pressure. Am J Emerg Med 1985; 3: 147-9.

15) Sanders AB, Kern KB, Otto CW, Milander MM, Ewy GA: End-tidal carbon dioxide monitoring during cardiopulmonary resuscitation. A prognostic indicator for survival. JAMA 1989; 262: $1347-51$.

16) Burnell JM, Scribner BH, Uyeno BT, Villamil MF: The effect in humans of extracellular $\mathrm{pH}$ change on the relationship between serum potassium concentration and intracellular potassium. J Clin Invest 1956; 35: 935-9.

17) Waters JH, Howard RS, Lesnik IK: Plasma potassium response after tromethamine (THAM) or sodium bicarbonate in the acidotic rabbit. Anesth Analg 1996; 83: 789-92.

18) Silver IA: Ion fluxes in hypoxic tissues. Microvasc Res 1977; 13: 409-20.

19) Kette F, Weil MH, Gazmuri RJ: Buffer solutions may compromise cardiac resuscitation by reducing coronary perfusion presssure. JAMA 1991; 266: 2121-6.
20) Graf H, Leach W, Arieff AI: Evidence for a detrimental effect of bicarbonate therapy in hypoxic lactic acidosis. Science 1985; 227: 754-6.

21) Delooz HH, Lewi PJ: Are inter-center differences in EMS-management and sodium-bicarbonate administration important for the outcome of CPR? The Cerebral Resuscitation Study Group. Resuscitation 1989; 17 Suppl: S161-72.

22) Roberts D, Landolfo K, Light RB, Dobson K: Early predictors of mortality for hospitalized patients suffering cardiopulmonary arrest. Chest 1990; 97: 413-9.

23) Stueven HA, Thompson B, Aprahamian C, Tonsfeldt DJ, Kastenson EH: Lack of effectiveness of calcium chloride in refractory asystole. Ann Emerg Med 1985; 14: 630-2.

24) Harrison EE, Amey BD: The use of calcium in cardiac resuscitation. Am J Emerg Med 1983; 1: 267-73.

25) Lindner KH, Ahnefeld FW, Pfenninger E: Arteriovenous pHand carbon dioxide gradients during cardiopulmonary resuscitation. Anaesthesist 1988; 37: 572-5. 